by adjusting the charge density of the $\mathrm{GO} / \mathrm{rGO}$ nanosheets. Thus, structures can be designed that exhibit both high ion diffusion and electron transport efficiency.

Additional work was conducted to explore the possibility of using this nanomaterial to increase the performance of supercapacitors. Graphene electrodes with an electric double layer (EDL) in supercapacitors have shown an increase in capacitance of ca. 100 farads per gram. More interesting, the overall capacitance was significantly enhanced by the hybridization of conductive graphene oxide nanosheets and the LDH nanosheets. This yielded a high capacitance of up to ca. 650 farads per gram, approximately six times that of pure graphene nanosheets. The direct combination of graphene with the insulating LDH nanosheets thus resulted in an improvement in the charge-transfer efficiency. Such a superfast charging and discharging performance potentially enables a huge energy output within a very short subsecond time scale.

According to the researchers, this work would be of benefit to applications in most electronic device and hybrid cars. Additionally, Ma said they expect that this three-dimensional transition metal/ graphene hybrid approach to be effective in developing non-noble metal electrocatalysis for applications such as fuel cells.

Jean. L. Njoroge
Feather microstructure leads to reduced friction surfaces

$\mathrm{T}$ he African darter duck is known to dive up to 35 meters without getting wet due to the microstructures of their feathers. Now, Michael Rubner, Gareth McKinley, and Robert E. Cohen from the Massachusetts Institute of Technology, Andrew Parker at the Natural History Museum, London, and their colleagues have correlated the birds' diving behavior with the microstructures of their wings. Their goal is to apply what they learn to create friction-reducing surfaces on water-going vessels.

By placing drops of different fluids on the birds' wings, the researchers found the "contact angle" to be very high. But those experiments were carried out in the open air. When ducks are immersed in water, the microstructures on their feathers entrain tiny pockets of air, forming an air film called a "plastron" which prevents water from wetting the feathers. Waterways, however, often contain an additional component: oils. Oils are known to wet bird feathers (observed as a low contact angle), which is why oil spills are particularly devastating to bird populations. Thus, before this technology can be successfully applied to ocean-going vessels, it is imperative to develop a mechanism for preventing oil from wetting the surfaces. As reported by the researchers in the July issue of the Journal of the Royal Society Interface (DOI: 10.1098/ rsif.2014.028), the key is replacement of the preening oils on feathers with a very low-energy fluorinated polymer composite, containing molecules known as fluorodecyl polyhedral oligomeric silsesquioxanes or F-POSS.

The coated duck wings also allowed the researchers to study the role of just the microstructures in the feathers' wetting behavior, essentially taking the variation of the ducks' preening oil out of the picture. This helped them understand the important contribution of larger scale defect structures that also are always present in feathers. In other words, the natural gaps in the duck feathers' micropattern prevent the duck feathers from staying dry to as great a diving depth as theory would predict. As a result, the plastron layer collapses and the feathers get wet beyond a certain diving depth.

However, the researchers' calculations show that feathers spontaneously dewet when the bird comes back up to the surface. The researchers speculate that there is a chance that the observed "wing spreading" behavior of birds such as cormorants helps them rapidly recover dry feathers after a deep dive. "I don't think you'll see ships that are able to "stretch out their wings,"' quipped McKinley, emphasizing that this work has augmented the understanding of the defect sensitivity of these superhydrophobic microstructures, a key to designing real surfaces that perform as desired.

Mary Nora Dickson

\section{Materials Researchers}

\section{C.N.R. Rao receives Bharat Ratna Award}

C.N.R. Rao, the Linus Pauling Research Professor and Honorary President at the Jawaharlal Nehru Centre for Advanced Scientific Research in Bangalore, received India's Bharat Ratna

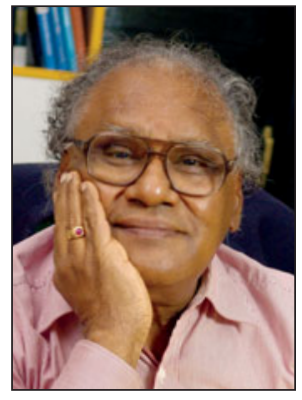

Award. This is India's highest civilian honor given for exceptional service toward advancement of art, literature, and science, and in recognition of public service of the highest order. Rao received the award from
President of India Pranab Mukherjee on February 4, 2014, in New-Delhi. Rao's research focused on solid-state and materials chemistry and on structural chemistry. He is the founder and president of the Materials Research Society of India. His service to his profession includes chair of the Science Advisory Council to the Prime Minister, and president of the Indian Academy of Sciences and of the Indian Science Congress. 